\title{
AVALIAÇÃO DA MATURIDADE EM GESTÃO DE PROJETOS EM UM DEPARTAMENTO DE DESENVOLVIMENTO DE NOVOS PRODUTOS
}

\section{ASSESSMENT OF PROJECT MANAGEMENT MATURITY IN A DEPARTMENT OF NEW PRODUCT DEVELOPMENT}

\author{
Pérsio Vitor de Sena Abrahão ${ }^{1}$; Edson Aparecida de Araujo Querido Oliveira ${ }^{2}$ \\ ${ }^{1}$ Universidade de Taubaté - UNITAU - Taubaté - SP - Brasil \\ persio.abrahao@terra.com.br \\ ${ }^{2}$ Universidade de Taubaté - UNITAU - Taubaté - SP - Brasil \\ edson.oliveira@unitau.com.br
}

\begin{abstract}
Resumo
Os projetos estão presentes no dia a dia de praticamente todas as organizações, com investimentos que representa quase um terço do PIB mundial. Muitas empresas têm utilizado os modelos de maturidade para avaliar a capacidade de gestão de seus projetos e estabelecer ações de melhoria. O Modelo Prado MMGP tem sido muito utilizado no Brasil para a avaliação de departamentos isolados. Ele, assim com a maioria deles, se baseia unicamente em respostas a um questionário, isso pode prejudicar a acurácia dos resultados em situações onde há uma quantidade muito reduzida de pessoas qualificadas para respondê-lo. Este estudo tem como objetivo verificar a aplicabilidade da análise dos documentos relacionados ao gerenciamento de projetos, como instrumento complementar ao método Prado-MMGP para ampliar as discussões e melhorar a avaliação do nível de maturidade. Para isso, optou-se pela realização de um estudo de caso único do tipo descritivo-exploratório em um departamento de desenvolvimento de novos produtos em uma empresa de base tecnológica de médio porte. Com a utilização de critérios semelhantes ao modelo MMGP pôde-se calcular os índices de aderência aos níveis e dimensões desse modelo, que produziram resultados comparáveis aos da aplicação do questionário e possibilitou analisar os resultados por diferentes fontes de evidência. Assim, pôde-se verificar que a utilização das evidências documentais pode trazer informações adicionais e contribuir para melhorar a acurácia na avaliação do nível de maturidade. Porém, sua aplicação fica restrita aos casos onde o pesquisador tenha acesso aos documentos dos projetos.
\end{abstract}

Palavras-chave: gerenciamento de projeto; modelo de maturidade; modelo MMGP.

\section{Introdução}

O gerenciamento de projetos não é algo novo. Desde a antiguidade, projetos como a construção das pirâmides no Egito ou a grande muralha na China necessitaram de esforços consideráveis para serem gerenciados. Entretanto, foi a partir da década de 1950 que muitos projetos militares de grande porte, conduzidos pelo governo dos Estados Unidos, no auge da Guerra 
Fria, passaram a demandar um novo tipo de organização de projetos e o desenvolvimento de ferramentas específicas de planejamento e controle (VALLE et al., 2010).

Essas ferramentas e técnicas de gestão estavam inicialmente restritas a poucas empresas ligadas aos projetos militares. A partir do final dos anos 1960, os executivos começaram a buscar novas técnicas de gerenciamento e estruturas organizacionais, motivados pela necessidade de se adaptarem às mudanças em um cenário cada vez mais competitivo. Com isso, inicia-se um forte crescimento e disseminação dos conhecimentos em gerenciamento de projetos (KERZNER, 2009).

No período pós-guerra até a década de 1980, denominado por Carvalho e Rabechini (2011) como embrionário, o gerenciamento de projetos ainda não tinha uma identidade e estava pulverizado em diversas áreas. Foi nesse período que surgiram as primeiras associações nos Estados Unidos e na Europa, o Project Management Institute (PMI) e a International Project Management Association (IPMA).

O período entre as décadas de 1980 e 1990 foi marcado por um crescimento exponencial do número de profissionais certificados, com foco na consolidação e disseminação das boas práticas. Foi na década de 1990 que várias associações publicaram as primeiras edições de seus guias de conhecimento, como o Project Management Body of Knowledge (PMBOK) em 1996 (CARVALHO; RABECHINI, 2011).

O surgimento dos modelos de maturidade em gerenciamento de projetos teve início nos anos 1990 em decorrência desse crescente interesse em melhorar o desempenho dos projetos no que se refere ao cumprimento dos prazos, controle de custos e qualidade (HERKENHOFF, 2010).

Carvalho e Rabechini (2011) denominam o período iniciado a partir dos anos 2000, como a segunda onda, com foco nos modelos organizacionais de gestão de projetos, quando as empresas passaram a investir de forma mais acentuada nestes modelos de maturidade, buscando a excelência em gerenciamento de projetos.

Segundo Prado (2010), os projetos representam atualmente quase um terço do PIB mundial e nenhuma empresa, independentemente de seu segmento ou estrutura, pode ignorar a importância de gerenciar de forma eficaz seus projetos.

O estudo feito pelo Standish Group (2013), avaliando projetos de TI, mostra que em 2012 apenas 39\% dos projetos foram concluídos com sucesso, $43 \%$ foram entregues com menos funcionalidades, com atrasos no cronograma e estouro de orçamento. Mostra ainda que o atraso médio foi de $74 \%$ e estouro de custo $59 \%$.

Estes números mostram a necessidade de se buscar formas de melhorar o gerenciamento dos projetos. Segundo Carvalho e Rabechini (2011) muitas empresas têm optado pela implantação de modelos de maturidade para gerir mudanças organizacionais que lhes permita administrar com maior eficácia esses empreendimentos. 
O Modelo Prado MMGP tem sido muito utilizado no Brasil para a avaliação da maturidade em departamentos isolados, tendo como diferencial o fator de disponibilizar uma base de dados aberta, com os resultados de pesquisas com empresas brasileiras, classificados por tipo, tamanho e segmento de atuação. Assim é possível realizar análises comparativas (benchmarking), que auxiliam no estabelecimento de metas de crescimento e posicionamento em relação ao setor específico.

Esse modelo, assim como a maioria deles, se baseia unicamente em respostas a um questionário. A acurácia dos resultados pode ser questionada em situações onde há uma quantidade muito reduzida de pessoas envolvidas com o gerenciamento de projetos que tenham condições de respondê-lo.

Este estudo teve como objetivo verificar a aplicação da análise documental dos projetos, como instrumento complementar ao método Prado-MMGP para o levantamento de evidências adicionais que pudessem ampliar as discussões e melhorar a avaliação do nível de maturidade em gerenciamento de projetos em um departamento de uma média empresa de base tecnológica.

Este artigo está organizado em cinco seções, conforme descrição resumida a seguir. A primeira contém a introdução ao tema abordado, ao problema e o objetivo do estudo. A segunda seção apresenta a metodologia utilizada, a terceira traz a revisão da literatura sobre o gerenciamento de projetos e os modelos de maturidade. Na quarta seção são apresentados os resultados e discussão do caso. E a quinta seção encerra o artigo com as considerações finais, seguida pelas referências bibliográficas utilizadas.

\section{Metodologia de Pesquisa}

Para este estudo optou-se por um estudo de caso do tipo descritivo-exploratório em um departamento de uma empresa de base tecnológica de médio porte, situada na Região Metropolitana do Vale do Paraíba (RMVALE).

A escolha da empresa teve como base as suas características e a acessibilidade aos documentos e informações dos projetos. O departamento avaliado foi constituído em 2009 com a missão conduzir os projetos de inovação, desenvolvendo equipamentos eletrônicos de alto conteúdo tecnológico, considerados estratégicos dentro do portfólio de produtos da empresa.

Como fonte de evidências para o estudo de caso foi utilizada a documentação de dois projetos desenvolvidos no período de 2012 a 2014, para fornecer detalhes específicos que pudessem corroborar os resultados da pesquisa de maturidade com a aplicação do modelo Prado MMGP. Foi elaborado um instrumento de coleta e análise que associa as evidências documentais aos temas propostos no questionário MMGP, versão 2.2.0. 
Com a utilização de critérios semelhantes aos propostos por Prado foi possível obter os índices de aderência aos níveis e dimensões do modelo MMGP. Assim foi possível comparar e discutir esses resultados com os a pesquisa pelo método Prado realizada anteriormente.

Segundo o método Prado, o cálculo do índice de aderência aos níveis 2, 3 e 4 é obtido pela somatória dos pontos nas dez questões do respectivo nível, as quais são respondidas utilizando uma escala de cinco alternativas, conforme mostra o Quadro 1. Para o nível 5 são utilizadas apenas duas opções, que correspondem às opções "a" e "e" respectivamente.

Quadro 1 - Escala de pontuação do método Prado MMGP

\begin{tabular}{|c|l|c|}
\hline OPÇÕES & \multicolumn{1}{|c|}{ CARACTERÍSTICAS } & PONTOS \\
\hline $\mathbf{a}$ & Aderência total a uma situação apresentada na opção “a”. & 10 \\
\hline b & $\begin{array}{l}\text { A situação existe, mas é levemente inferior ao apresentado na opção } \\
\text { "a”. }\end{array}$ & 7 \\
\hline c & $\begin{array}{l}\text { A situação existe, mas é significativamente inferior ao apresentado na } \\
\text { opção “a”. }\end{array}$ & 4 \\
\hline d & $\begin{array}{l}\text { Esforços foram iniciados em relação à situação apresentada na opção } \\
\text { "a". }\end{array}$ & 2 \\
\hline e & Nenhum esforço foi iniciado nesse sentido. & 0 \\
\hline
\end{tabular}

Fonte: Prado (2010)

A partir do instrumento de coleta e análise de evidências documentais são calculados os índices de aderências aos níveis, os quais podem ser comparados com os valores médios obtidos com a pesquisa MMGP. As eventuais divergências apontam a necessidade de um maior aprofundamento da análise na questão específica. Os índices obtidos de aderências aos níveis são interpretados utilizando a escala apresentada na Figura 1, conforme proposto no modelo Prado (2010).

Figura 1 - Escala de pontuação do Índice de Aderência aos Níveis (IAN)

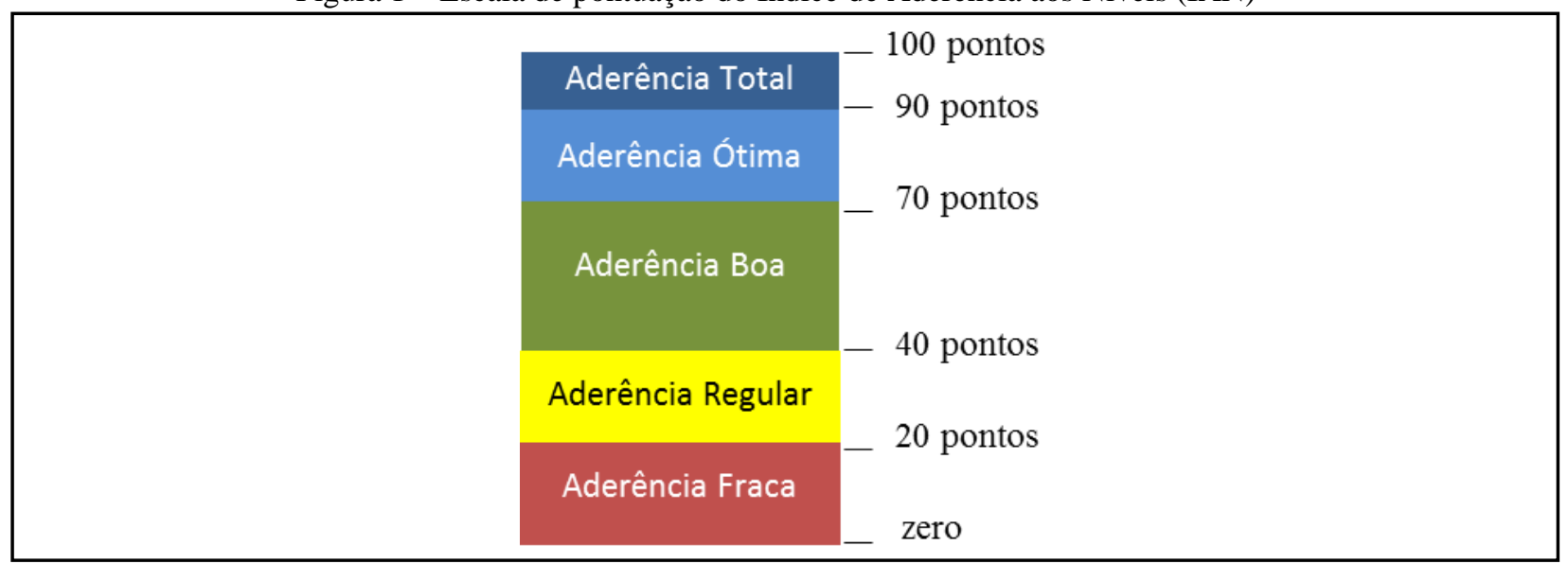

Fonte: Prado (2010) 
As análises de aderência às dimensões MMGP são realizadas com base nos percentuais de aderência a partir das três bases de dados. Os percentuais de aderência calculados a partir das análises de evidências são realizados utilizando critérios semelhantes aos propostos por Prado e servem de referência para analisar os dados da pesquisa MMGP.

O percentual de aderência a uma determinada dimensão do modelo Prado-MMGP corresponde à relação entre a somatória dos pontos obtidos nas questões correlacionadas a essa dimensão e a pontuação máxima, sendo obtida pela Equação (1).

\begin{tabular}{|c|c|c|c|c|}
\hline \multirow{2}{*}{$\begin{array}{l}\text { Aderência à } \\
\text { Dimensão }\end{array}$} & $=$ & $\Sigma$ pontuação obtida & \multirow{2}{*}{ x $100 \%$} & \multirow{2}{*}{ Equação (1) } \\
\hline & & Pontuação Máxima & & \\
\hline
\end{tabular}

A Avaliação Final de Maturidade (AFM) é determinada pela soma dos pontos obtidos nos diversos níveis e corresponde a uma média entre os percentuais de aderência a esses níveis, calculada pela Equação (2).

$\mathrm{AFM}=\frac{100+\Sigma \text { pontuação obtida }}{100} \quad$ Equação (2)

O cálculo da avaliação final de maturidade (AFM) do departamento é efetuado com base nos resultados de aderências aos níveis, após a análise conjunta da pesquisa MMGP e análise documental dos projetos.

O índice de avaliação final (AFM) indica de forma quantitativa o grau de maturidade em gerenciamento de projetos pelo método Prado MMGP. A interpretação do valor obtido é feita com base na escala de cinco pontos, correspondentes aos cinco níveis do modelo, conforme a Figura 2.

Figura 2 - Escala de pontuação da Avaliação Final de Maturidade (AFM)

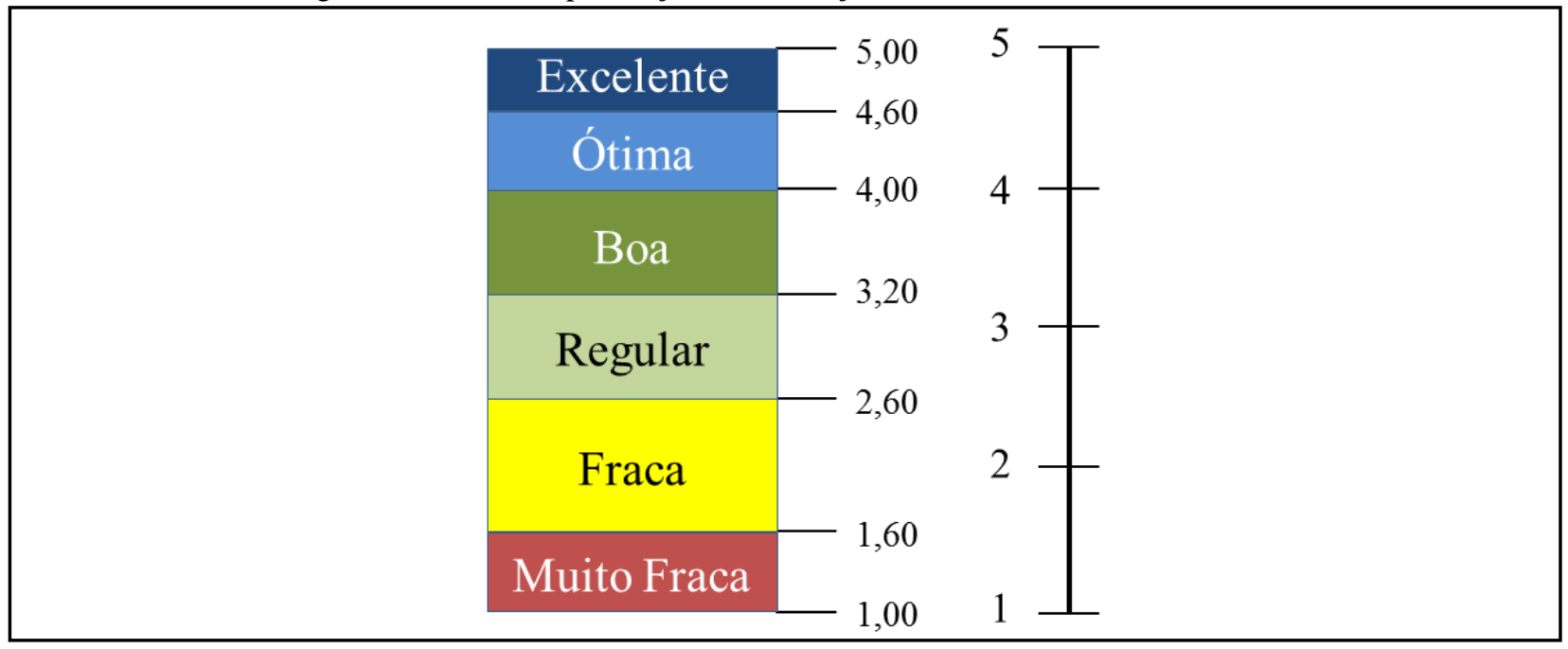

Fonte: Prado (2010) 


\section{Revisão da Literatura}

\subsection{Gerenciamento de Projetos}

As diversas técnicas e ferramentas desenvolvidas para o gerenciamento dos grandes projetos militares, conduzidos pelos Estados Unidos na década de 1950, passaram a ser incorporadas por inúmeras empresas, incentivadas, segundo Valle et al. (2010), pela: (1) Reengenharia, que busca eficiência e eliminação de atividades sem valor agregado; (2) Globalização, a crescente interdependência econômica entre os países, com o aumento de interação entre pessoas e equipes em empresas transnacionais; e (3) Informatização, que facilitou o acesso dos gerentes de projetos às ferramentas como CPM e PERT (VALLE et al., 2010).

Maximiano (2014) utiliza a designação administração de projeto para o gerenciamento de projeto e define como o processo de tomar decisões para realizar atividades temporárias, com o objetivo de fornecer um resultado. A administração de projetos envolve a aplicação de técnicas visando alcançar um determinado resultado, dentro das restrições de tempo e custo, em que a tarefa básica é assegurar a orientação do esforço para o resultado, controlando custos, prazos e riscos.

Kerzner (2009) adota uma definição de gerenciamento de projeto com foco no planejamento e controle. Para ele, esse gerenciamento envolve o planejamento, organização, direção e controle dos recursos da empresa para atingir um objetivo de curto prazo, e a abordagem caracteriza-se pela adoção de técnicas especiais de gerenciamento com o propósito de obter melhor controle e utilização dos recursos existentes.

O PMI (2013a) adota uma definição semelhante à de Kerzner, porém com um foco na aplicação dos conhecimentos, técnicas e habilidades. O gerenciamento de projeto é a aplicação dos conhecimentos, técnicas, ferramentas e habilidades às atividades do projeto para atender aos seus requisitos, e normalmente inclui:

- Identificação dos requisitos;

- Gerenciamento das diferentes necessidades e expectativas das partes interessadas;

- Gestão eficaz e colaborativa da comunicação entre as partes interessadas; e

- Equilíbrio das restrições conflitantes do projeto: escopo, qualidade, cronograma, orçamento, recursos, e riscos, entre outros.

O PMI (2013a, p.5) define o gerenciamento de projetos como a "aplicação do conhecimento, habilidades, ferramentas e técnicas às atividades do projeto para atender aos seus requisitos”. Esse gerenciamento realiza-se pela aplicação e integração apropriada dos 47 processos em dez áreas de conhecimento, agrupados em cinco grupos, que são:

O PMI (2013a, p.5) define o gerenciamento de projetos como a "aplicação do conhecimento, habilidades, ferramentas e técnicas às atividades do projeto para atender aos seus requisitos". Esse 
gerenciamento realiza-se pela aplicação e integração apropriada dos 47 processos em dez áreas de conhecimento, agrupados em cinco grupos, que são:

- Processos de iniciação: conjunto de processos executados para definir um novo projeto ou nova fase de um projeto;

- Processos de planejamento: conjunto de processos necessários à elaboração do Plano de Gerenciamento do Projeto, que define as ações necessárias para alcançar seus objetivos;

- Processos de execução: processos realizados para executar o trabalho definido no Plano de Gerenciamento do Projeto;

- Processos de monitoramento e controle: processos exigidos para acompanhar, analisar e controlar o progresso e desempenho do projeto, conforme as linhas de base estabelecidas no Plano de Gerenciamento do Projeto; e

- Processos de encerramento: processos necessários para finalizar todas as atividades do projeto, visando encerrar formalmente o projeto ou fase.

\subsection{Maturidade em Gerenciamento de Projetos}

Nas últimas décadas tem ocorrido uma crescente preocupação, por parte das organizações e da comunidade científica, com o nível de maturidade das organizações em gerenciamento de projetos visando aumentar a probabilidade de sucesso dos projetos.

Em uma publicação do PMI, Cartwright e Yinger (2007) afirmam que existe uma relação positiva entre o sucesso de um projeto e a competência do gerente do projeto; a maturidade organizacional e outros fatores contingenciais que o envolvem, tais como o tipo, as características do ciclo de vida, e a estrutura do projeto.

Segundo Prado (2010), o conceito de maturidade é bastante intuitivo e com aplicações em vários aspectos dos dia a dia. No caso de gerenciamento de projetos, a maturidade está associada à capacidade da organização em gerenciá-los.

Kerzner (2009, p. 59) define "a excelência de uma organização no gerenciamento de projetos como a sua capacidade de criar um ambiente no qual haja um fluxo contínuo de projetos gerenciados com sucesso". A maturidade em gerenciamento de projetos como a implantação de uma metodologia padronizada e processos de acompanhamento que aumentem a probabilidade de sucessos repetidos, que implica na existência de ferramentas, técnicas, processos e até mesmo uma cultura adequada.

O PMI (2013b) apresenta uma visão evolutiva e multidimensional de maturidade, incluindo processos eficazes bastante diversos como planejamento estratégico de negócios, desenvolvimento de negócios, engenharia de sistemas, gestão de projetos, gestão de riscos, tecnologia da informação (TI), ou gestão de pessoas. 
Definir o que vem a ser o sucesso de um projeto não é uma tarefa muito simples, pois depende da perspectiva da parte interessada, do tipo de projeto, da componente temporal e do foco da análise, ou seja, o foco pode estar no gerenciamento do projeto, no produto gerado, ou nos resultados obtidos.

Para Carvalho e Rabechini (2011), o conceito de sucesso está relacionado à perspectiva de tempo, quando no curto prazo o sucesso, o foco principal, está no gerenciamento do projeto, na entrega do resultado dentro do prazo, do orçamento e com a qualidade acordada. Em uma perspectiva de médio prazo o foco está no cliente e no desempenho do produto, enquanto que em longo prazo está nos benefícios proporcionados pelo projeto.

O PMBOK (PMI, 2013a, p. 16) apresenta três perspectivas de sucesso. Na primeira, com foco no projeto, “o sucesso é medido pela qualidade do produto e do projeto, pela pontualidade, pelo cumprimento do orçamento e pelo grau de satisfação do cliente". Na segunda o foco está no programa, com o "sucesso medido pelo grau em que o programa atende às necessidades e pelos benefícios para os quais foi executado". A terceira perspectiva tem foco no portfólio, o sucesso "é medido em termos do desempenho de investimento agregado e realização dos benefícios do portfólio".

Segundo Vargas (2005), o projeto bem-sucedido é aquele que foi realizado conforme o planejado. É importante que seja concluído dentro do tempo e orçamento previstos e que tenha: utilizado os recursos de forma eficiente; atingido a qualidade e desempenho desejado; sido aceito sem restrições pelo cliente; e não tenha agredido a cultura da organização.

A definição de Vargas (2005) está alinhada com o PMBOK na perspectiva do projeto e com a definição de Carvalho e Rabechini (2011) na perspectiva do gerenciamento do projeto.

Gráfico 1 - Relação entre utilização de metodologia de GP e sucesso em projetos

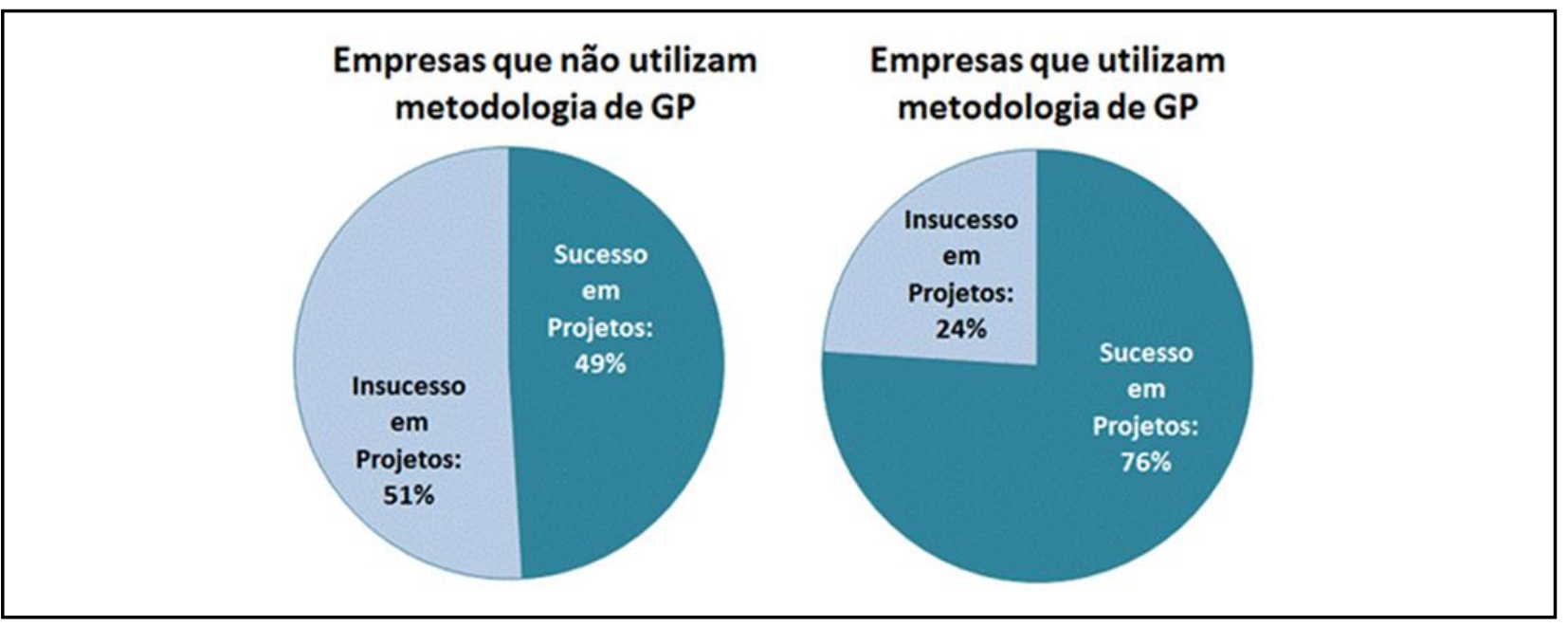

Fonte: PMSURVEY (2008) 
De acordo com o estudo de benchmarking PMSURVEY (2008) as empresas que utilizavam uma metodologia de gerenciamento de projetos apresentavam um percentual de sucesso em seus projetos significativamente maior do que aquelas que não utilizavam, sugerindo uma relação direta entre essas variáveis, conforme mostram o Gráfico 1.

\subsection{Modelos de Maturidade}

Os modelos de maturidade visam avaliar a capacidade de a organização gerenciar seus projetos de forma eficiente e eficaz. Sua maior contribuição está na identificação do nível atual de maturidade e na estruturação de ações para o desenvolvimento de novas capacidades, aumentando assim a probabilidade de sucesso de seus projetos (SANTOS, 2009). Para Thielmann e Silva (2014), apesar de existirem vários modelos de maturidade, os três mais citados na literatura são:

- Kerzner PMMM: Project Management Maturity Model;

- PMI OPM 3: Organizational Project Management Maturity Model (OPM3); e

- Prado: Modelo de Maturidade em Gestão de Projetos (MMGP).

\subsubsection{Modelo SEI-CMM}

O Capability Maturity Model (CMM) foi o primeiro modelo de maturidade, lançado em agosto de 1991. Ele foi desenvolvido pela Universidade Carnegie Mellon em parceria com o System Engineering Institute, com o objetivo de auxiliar o departamento de defesa americano na escolha de fornecedores de software, tomando como base as atitudes gerenciais encontradas nas empresas no processo de desenvolvimento de software (HERKENHOFF, 2010).

Figura 3 - Níveis de maturidade do CMM

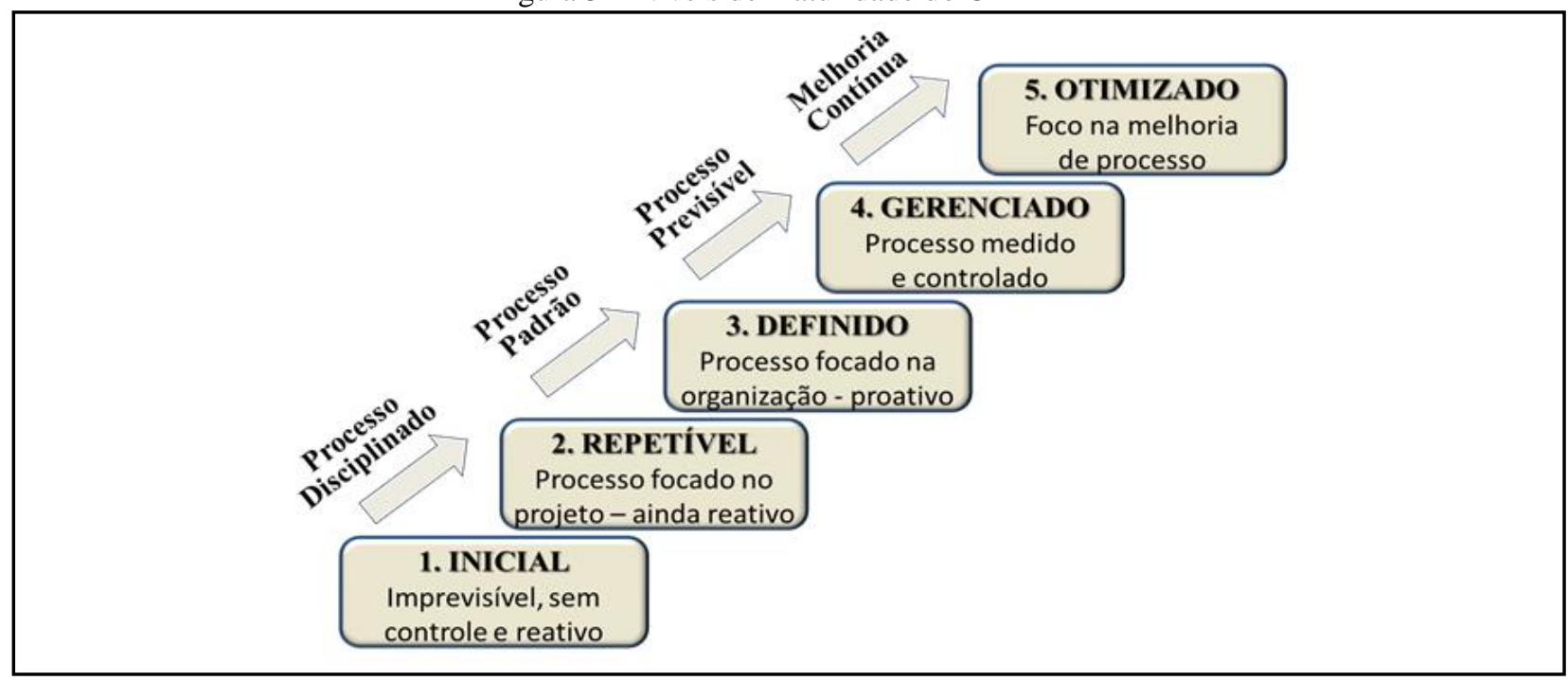

Fonte: Adaptado de Carvalho et al (2005) 
A Figura 3 apresenta os níveis de maturidade do modelo CMM, descritos a seguir. Cada um dos cinco níveis corresponde a um conjunto de áreas chaves de processo (MEZZENA; ZWICKER, 2007).

- Nível 1 - Inicial: caracteriza-se pela informalidade do processo de desenvolvimento de software, caótico e com ações reativas, e frequentemente ultrapassa os prazos e custos dos projetos;

- Nível 2 - Repetível: os processos básicos de gerenciamento de projetos para o acompanhamento de cronograma, custos e das funcionalidades estão implantados, permitindo repetir os sucessos anteriores em projetos similares;

- Nível 3 - Definido: caracteriza-se pela existência de processo integrado padrão para a organização, em que os procedimentos de gestão de projetos e atividades de engenharia estão documentos e são utilizados de forma padronizada para as atividades de desenvolvimento e manutenção;

- Nível 4 - Gerenciado: utiliza medições detalhadas do processo de desenvolvimento e qualidade dos produtos, que são analisados e controlados de forma quantitativa; e

- Nível 5 - Otimizado: caracteriza-se pela implantação de um processo de melhoria contínua, por meio da retroalimentação quantitativa e análises comparativas, em um processo na busca de novas ideias e tecnologias inovadoras.

\subsubsection{Modelo Kerzner PMMM}

O modelo denominado Project Management Maturity Model (PMMM), proposto por Kerzner, foi apresentado no congresso do PMI em 1999, publicado pela primeira vez em 2001 e ajustado e reeditado em 2005 como objetivo de analisar a maturidade em gerenciamento de projetos nas empresas por meio de cinco níveis e conceitos similares ao CMM (OLIVEIRA, 2013).

O modelo Kerzner-PMMM se diferencia do CMM em vários aspectos. Esses modelos utilizam terminologias distintas, que segundo Carvalho, Laurindo e Pessoa (2003) podem levar a alguns problemas quando implementados simultaneamente.

Carvalho et al (2005) afirmam ainda que há uma complementariedade entre eles e a possibilidade de sinergia mútua. Um dos pontos fortes é a utilização das dez áreas de conhecimento descritas no PMBOK nos diversos níveis de maturidade.

Para Kerzner (2001, p.42), o PMMM “é um modelo que descreve os fundamentos para se atingir a excelência em gerenciamento de projetos, composto por cinco níveis", conforme mostra a Figura 4. 
Figura 4 - Cinco níveis do modelo de maturidade Kerzner-PMMM

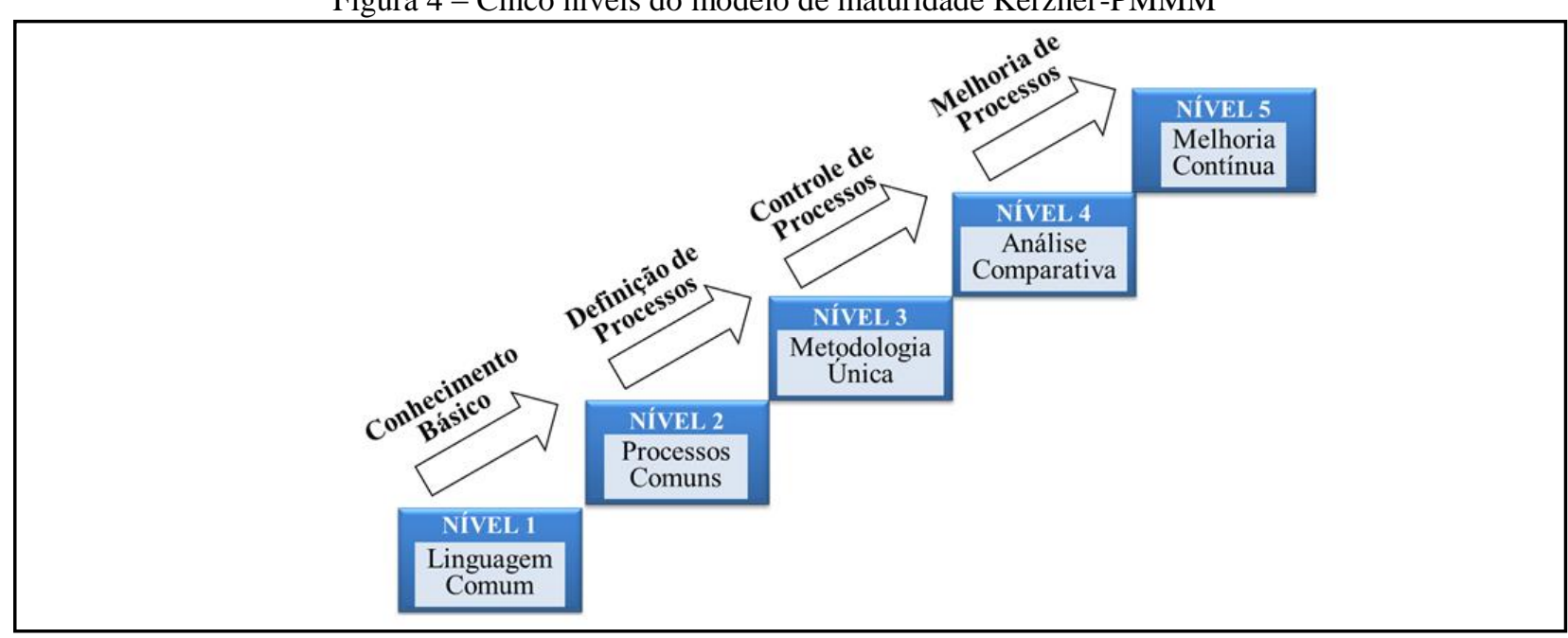

Fonte: adaptado de Kerzner (2001)

\subsubsection{Modelo Prado-MMGP}

Prado (2010) propôs dois modelos de maturidade em gerenciamento de projetos. O primeiro é o modelo setorial ou departamental, MMGP-setorial, lançado em 2002 para a avaliação de setores isolados de uma organização. Já o segundo, o modelo corporativo, foi lançado em 2004 e desenvolvido para avaliação global do nível de maturidade da organização, envolvendo, além dos setores diretamente ligados aos projetos, os setores corporativos.

Desde 2005, tem sido realizada, com apoio dos capítulos do PMI e International Project Management Association (IPMA), a pesquisa de maturidade em gerenciamento de projetos com a aplicação desse modelo em diversas empresas. A Pesquisa 2014 envolveu 7.885 projetos em 415 empresas do setor privado, público e terceiro setor (PRADO; OLIVEIRA, 2015).

Autores como Herkenhoff (2010) e Santos (2010) ressaltam a grande utilização desse modelo na avaliação de organizações brasileiras, com resultados condizentes com a realidade, que segundo eles atestam sua credibilidade.

Figura 5 - Níveis e dimensões do modelo Prado - MMGP

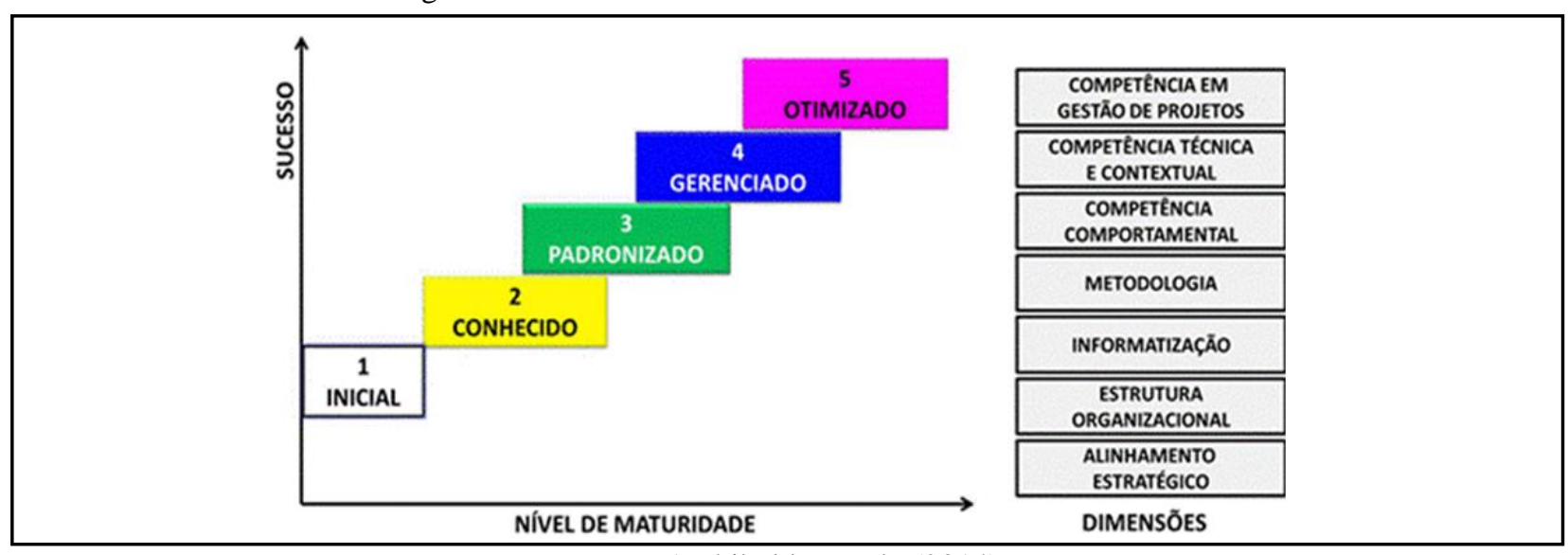

Fonte: Archibald e Prado (2014) 
O Modelo Setorial está estruturado em cinco níveis e sete dimensões, conforme mostra a Figura 5, contemplando estratégias, processos pessoas, tecnologia e ferramentas com linguagem aderente à terminologia do PMBOK (PMI, 2013).

Os cinco níveis de maturidade do modelo Prado-MMGP, descritos por Prado (2010) são:

- Nível 1 - Inicial ou Embrionário: não há uma percepção correta do que seja gerenciamento de projetos. A execução é feita com base na intuição, "boa vontade" ou "melhor esforço" individual. O sucesso do projeto depende do esforço individual e é grande a probabilidade de atraso, estouro de orçamento, e não atendimento do escopo e especificações técnicas, pois normalmente não se faz planejamento e o controle é inexistente;

- Nível 2 - Conhecido: há um despertar da consciência quanto à importância do gerenciamento de projetos, com investimento em treinamento e aquisição de softwares específicos, porém, não há uma padronização de procedimentos e sim iniciativas isoladas de uso dos conhecimentos e ferramentas de sequenciamento de atividades;

- Nível 3 - Padronizado: há uma estrutura organizacional adequada e uma metodologia disponível, parcialmente informatizada, com procedimentos padronizados praticados por todos, além de uma evolução das competências técnicas, comportamentais e contextuais dos gerentes de projetos, com uma significativa melhoria no desempenho dos projetos (prazo, custo, escopo e qualidade), porém os problemas ainda não foram sanados;

- Nível 4 - Gerenciado: os processos implantados anteriormente foram consolidados e as anomalias resolvidas, a partir da eliminação ou mitigação das causas dos desvios que atrapalham os resultados dos projetos. O ciclo de melhoria contínua é aplicado sempre que alguma deficiência for detectada e há um forte alinhamento dos projetos aos negócios da organização. Os gerentes demonstram um alto nível de competência, alinhando conhecimento e experiência. Há um alto índice de sucesso, compatível com o esperado para esse nível de maturidade; e

- Nível 5 - Otimizado: existência de uma plataforma padronizada de GP que funciona e dá resultados. Os processos de planejamento e execução foram otimizados, melhorando o desempenho dos projetos. A otimização é feita com base nos conhecimentos, larga experiência, atitudes pessoais, como disciplina e liderança, entre outras; e um excelente banco de dados das melhores práticas. Uma quantidade significativa de projetos utilizou a metodologia com alto nível de sucesso, e a organização aceita desafios de alto risco dada a confiança em seus profissionais.

O modelo MMGP define cinco níveis e sete dimensões para avaliar grau de maturidade, e cada nível pode conter até sete dimensões com diferentes peculiaridades As sete dimensões, apresentadas a seguir, formam a plataforma para gestão de projetos (ARCHIBALD; PRADO, 2014). 
- Competência em Gerenciamento de Projetos e de Programas: os principais envolvidos na gestão dos projetos devem possuir experiência e conhecimento das técnicas e boas práticas de gerenciamento de projetos. O Guia PMBOK do PMI (2013a), e o ICB (IPMA Competence Baseline) são referências relevantes sobre este tema;

- Competência Técnica e Contextual: os principais envolvidos na gestão dos projetos devem ser experientes e com conhecimento técnico relacionado ao produto ou serviço, bem como aspectos da organização, seu modelo produtivo, gestão financeira e mercados;

- Competência Comportamental: os principais envolvidos na gestão dos projetos devem possuir conhecimento e habilidades nos aspectos de comunicação, liderança, motivação, negociação entre outros;

- Uso de Metodologia: deve haver uma metodologia de gerenciamento de projetos que contemple todo o ciclo de vida do projeto, incluindo a fase de análise do negócio;

- Informatização: os aspectos relevantes da metodologia devem ser suportados por um sistema informatizado que facilite o processo de planejamento, controle e tomadas de decisão;

- Alinhamento Estratégico: os projetos executados pelo setor devem estar alinhados à estratégia da organização; e

- Estrutura Organizacional: a estrutura organizacional deve ser adequada à implementação dos projetos. Geralmente essa estrutura inclui a presença de gerentes de projetos, patrocinador, comitês, escritório de projetos (EGP), bem como a definição dos papeis, funções, regras e relação de autoridade e poder entre as diversas áreas.

Prado (2010) relaciona o amadurecimento da organização com o sucesso de seus projetos e define a maturidade em gerenciamento de projetos como a sua capacidade de gerenciá-los.

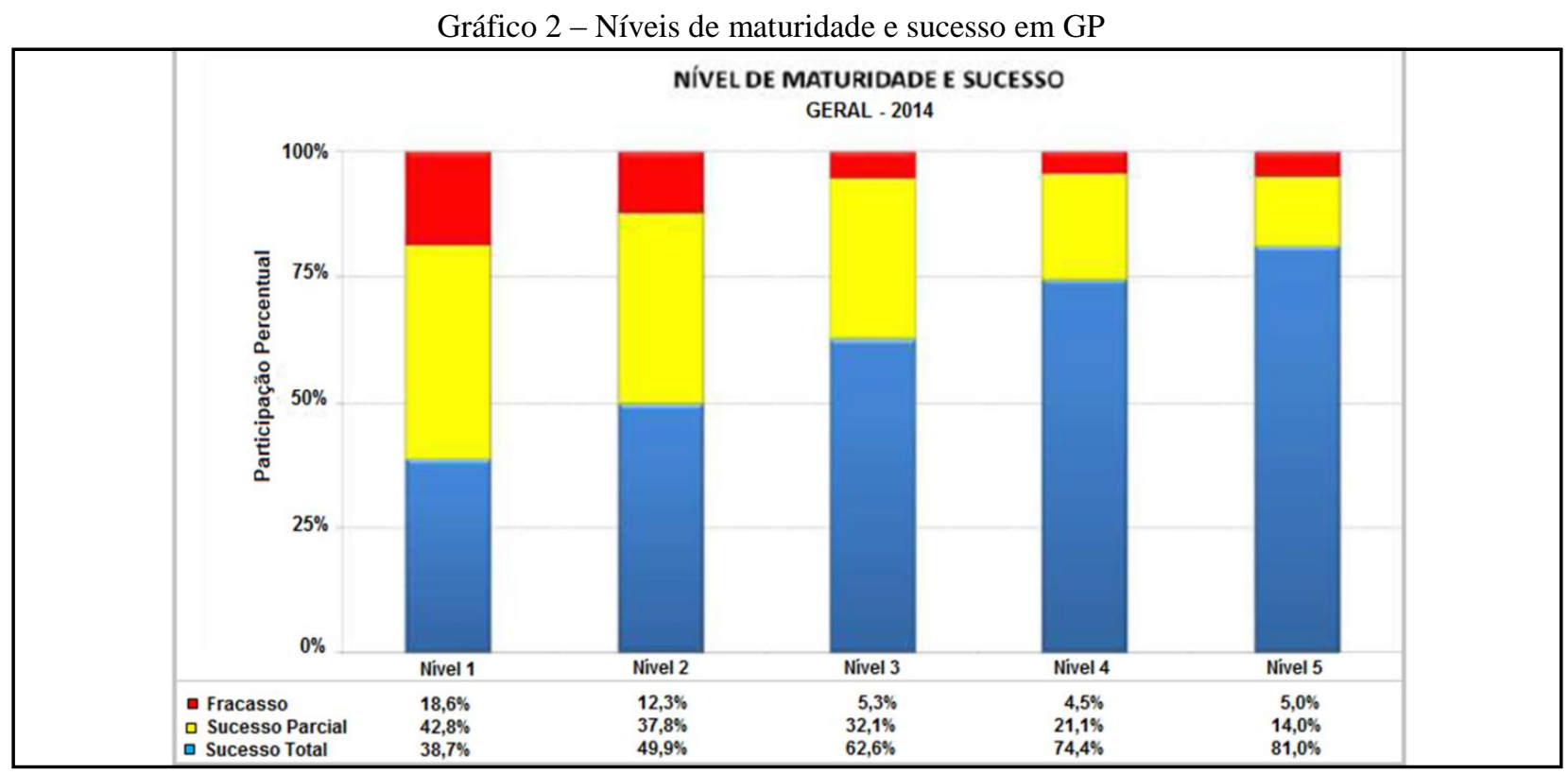

Fonte: Prado e Oliveira (2012) 
O Gráfico 2 apresenta o resultado da pesquisa de Prado e Oliveira (2015), que envolveu 7.885 projetos de organizações brasileiras de diversos setores. Esse gráfico evidencia que há uma relação direta entre os níveis de maturidade das organizações e os resultados dos projetos, ou seja, quanto maior o nível de maturidade, maior o percentual de sucesso dos projetos.

Nesse trabalho, o conceito de sucesso está relacionado à capacidade da organização em concluir o projeto dentro do prazo, do orçamento, e com a qualidade acordada.

\section{Resultados e Discussão}

O departamento estudado foi fundado em 2009 com a missão de desenvolver tecnologias e produtos inovadores para modernizar e ampliar a linha de produtos da empresa e obteve o apoio da Finep, no programa de subvenção econômica para vários projetos.

No período de 2012 a 2014, o departamento contava com uma equipe multidisciplinar, composta por 25 funcionários, na sua maioria engenheiros, sendo nove deles com titulação de mestrado nas áreas de atuação. Esta equipe estava organizada em grupos de especialistas e os projetos eram executados em uma estrutura matricial.

A empresa é certificada ISO 9001 e tem uma cultura com foco na qualidade e na organização por processos. Os produtos desenvolvidos têm aplicação aeronáutica, sendo necessário que sejam certificados antes de se tornarem comerciais.

O departamento de desenvolvimento de novos produtos foi estabelecido dentro deste contexto e pode-se constatar a existência de processos detalhados e bem documentados, referentes ao desenvolvimento de produtos, gerenciamento de requisitos, análises da qualidade, controles de configuração e mudanças, entre outros. Mas perceber-se a falta de processos específicos de gerenciamento de projetos.

Os dois projetos selecionados neste estudo tiveram início em 2012 com previsão inicial de encerramento em 2014, porém, ao final dos 36 meses os orçamentos estavam estourados e seriam necessários mais 6 meses para concluí-los.

\subsection{Análise da Aderência aos Níveis e Dimensões do Modelo}

O índice de aderência ao nível 5 não foi calculado, sendo considerado igual a zero, uma vez que, o departamento é muito jovem, com pouca experiência em gerenciamento de projetos e que as respostas na avaliação desse nível têm apenas duas opções, atendimento pleno ou não atende, a avaliação esperada seria de não atendimento pleno em nenhuma das dimensões do modelo.

Os índices de aderência aos demais níveis de maturidade em gerenciamento de projetos desse departamento foram calculados a partir da análise documental dos projetos em comparação com os resultados da pesquisa MMGP, que teve a participação de sete profissionais do 
departamento, sendo três destes com função gerencial e quatro com função de coordenação técnica dos projetos, conforme mostra a Tabela 1.

Tabela 1 - Índices de Aderências aos Níveis por participante da Pesquisa MMGP

\begin{tabular}{c|c|c|c|c|c|c|c}
\hline \multirow{2}{*}{$\begin{array}{c}\text { QUESTÕES } \\
\text { MMGP } \\
\text { vers. 2.2.0 }\end{array}$} & Ger_1 & Ger_2 & Ger_3 & Coord_1 & Coord_2 & Coord_3 & Coord_4 \\
\cline { 2 - 8 } & 50 & 39 & 49 & 18 & 29 & 69 & 34 \\
\hline IAN 2: & 18 & 16 & 30 & 10 & 8 & 29 & 12 \\
\hline IAN 3: & 34 & 24 & 28 & 10 & 26 & 20 & 14 \\
\hline IAN 4: & 0 & 0 & 0 & 0 & 0 & 0 & 0 \\
\hline IAN 5: & $\mathbf{1 0 2}$ & $\mathbf{7 9}$ & $\mathbf{1 0 7}$ & $\mathbf{3 8}$ & $\mathbf{6 3}$ & $\mathbf{1 1 8}$ & $\mathbf{6 0}$ \\
\hline $\boldsymbol{\Sigma}$ & $\mathbf{2 , 0 2}$ & $\mathbf{1 , 7 9}$ & $\mathbf{2 , 0 7}$ & $\mathbf{1 , 3 8}$ & $\mathbf{1 , 6 3}$ & $\mathbf{2 , 1 8}$ & $\mathbf{1 , 6 0}$ \\
\hline AFM
\end{tabular}

Os resultados dos índices de aderência aos níveis a partir dos dados da pesquisa MMGP apresenta uma dispersão muito grande se considerada a média geral com todos os respondentes, conforme mostra coeficiente de variação (CV) na Tabela 2, que expressa a relação percentual do desvio padrão em relação à média.

Tabela 2 - Índices de Aderências aos Níveis por participante da Pesquisa MMGP

\begin{tabular}{|c|c|c|c|c|c|c|c|c|c|c|}
\hline \multirow{3}{*}{$\begin{array}{c}\text { QUESTÕES } \\
\text { MMGP } \\
\text { vers. 2.2.0 }\end{array}$} & \multirow{3}{*}{$\begin{array}{c}\text { AVALIAÇÃO } \\
\text { DE } \\
\text { EVIDÊNCIAS }\end{array}$} & \multicolumn{9}{|c|}{ PARTICIPANTES DA PESQUISA MMGP } \\
\hline & & \multicolumn{3}{|c|}{$\begin{array}{l}\text { GERAL: Todos os } \\
\text { Participantes }\end{array}$} & \multicolumn{3}{|c|}{$\begin{array}{l}\text { GRUPO 1: } \\
\text { Gestores }\end{array}$} & \multicolumn{3}{|c|}{$\begin{array}{c}\text { GRUPO 2: } \\
\text { Coordenadores } \\
\end{array}$} \\
\hline & & MÉDIA & $\begin{array}{l}\text { Desvio } \\
\text { Padrão }\end{array}$ & CV & MÉDIA & $\begin{array}{l}\text { Desvio } \\
\text { Padrão }\end{array}$ & CV & MÉDIA & $\begin{array}{l}\text { Desvio } \\
\text { Padrão }\end{array}$ & CV \\
\hline IAN 2: & 49 & 41,1 & 16,6 & $40 \%$ & 46,0 & 6,1 & $13 \%$ & 37,5 & 22,0 & $59 \%$ \\
\hline IAN 3: & 22 & 17,6 & 8,8 & $50 \%$ & 21,3 & 7,6 & $36 \%$ & 14,8 & 9,6 & $65 \%$ \\
\hline IAN 4: & 23 & 22,3 & 8,3 & $37 \%$ & 28,7 & 5,0 & $17 \%$ & 17,5 & 7,0 & $40 \%$ \\
\hline IAN 5: & 0 & 0,0 & 0,0 & & 0,0 & 0,0 & & 0,0 & 0,0 & \\
\hline$\Sigma$ & 94 & 81 & & & 96 & & & 69,8 & & \\
\hline AFM & 1,94 & 1,81 & & & 1,96 & & & 1,70 & & \\
\hline
\end{tabular}

O cálculo dos índices de aderência aos níveis utilizando a análise de evidências documentais traz o mesmo tipo de informação a partir de uma segunda fonte de evidências, que permite comparar com os resultados obtidos a partir da aplicação do questionário.

A Tabela 2 permite comparar os resultados da análise de evidências documentais com os resultados da pesquisa, estratificados em dois grupos de participantes, o primeiro, denominado Grupo 1, formado pelos três participante com funções gerenciais, o Grupo 2 com os demais participantes e compará-los aos resultados. 
A comparação dos valores médios dos índices de aderência aos níveis dos Grupos 1 e 2 com os resultados da avaliação de evidências ressalta a discrepância dos resultados do Grupo 2. O Grupo 1 apresenta valores médios mais próximos aos da avaliação de evidências e coeficientes de variação significativamente inferiores.

O Gráfico 3 apresenta os percentuais de aderência às sete dimensões do modelo prado, comparando os valores calculados a partir das análises de evidências documentas (linha pontilhada) com os resultados da pesquisa MMGP para o subgrupo 1 - Gestores (linha azul) e o Grupo 2 Coordenadores (linha vermelha).

Gráfico 3 - Percentuais de Aderência às Dimensões do Modelo MMGP

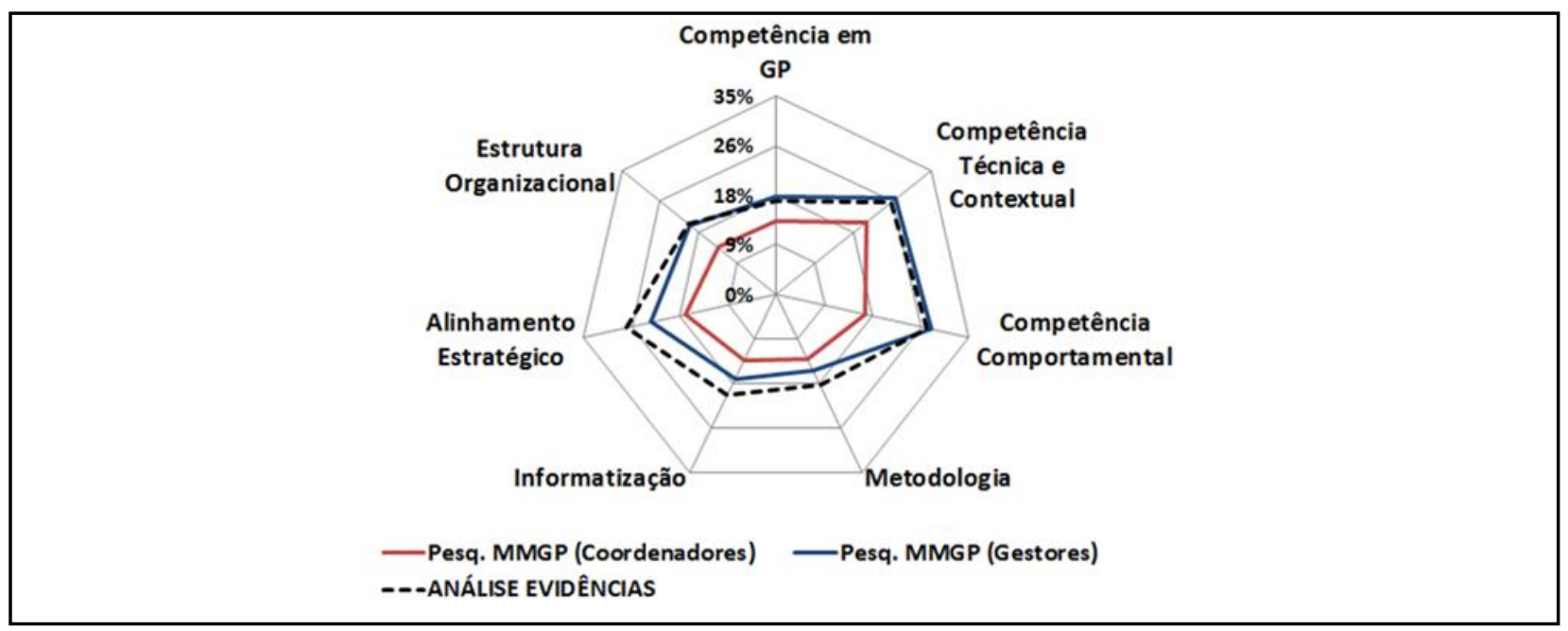

As discrepâncias dos resultados da pesquisa MMGP com os dados do Grupo 2 podem indicar que estes coordenadores têm uma visão limitada do tema gestão de projetos e/ou dos processo envolvidos. Assim, este grupo não seria elegível para participarem da pesquisa, conforme recomendado por Prado (2010).

\subsection{Avaliação Final de Maturidade - AFM}

A Avaliação Final de maturidade do departamento estudado foi determinada a partir das análises de evidências e representa uma média dos índices de aderência aos diversos níveis, calculada pela equação (2), apresentada na seção 2 deste artigo.

O Gráfico 4 apresenta os níveis de aderência aos níveis do modelo Prado-MMGP e o valor da Avaliação Final da Maturidade do departamento que ficou com 1,94, considerado Fraco, com um Índice de Aderência ao Nível 2 abaixo de 50 pontos e próximo de 20 nos níveis três e quatro. 


\begin{tabular}{|c|c|c|c|c|c|c|c|c|c|c|c|}
\hline \multicolumn{12}{|c|}{ Avaliação Final da Maturidade: 1,94} \\
\hline Níveis & Pontos & 10 & 20 & 30 & 40 & 50 & 60 & 70 & 80 & 90 & 100 \\
\hline 2 & 49 & & & & & & & & & & \\
\hline 3 & 22 & & & & & & & & & & \\
\hline 4 & 23 & & & & & & & & & & \\
\hline 5 & 0 & & & & & & & & & & \\
\hline
\end{tabular}

Essa avaliação, AFM = 1,94, posiciona o departamento estudado no Nível 1 - INICIAL, em um estágio próximo de atingir o Nível 2 - CONHECIDO. Nesse nível, os processos ainda estão estabelecidos e o gerenciamento de projetos é executado com base na intuição e esforço individual.

Existe a utilização de softwares para elaboração e controle de cronogramas de forma não padronizado e incipiente. Já há um despertar para a importância da utilização de processos padronizados visando facilitar o planejamento e controle.

Ao comparar esse resultado com as empresas nacionais de Desenvolvimento de Novos Produtos (DNP), que na pesquisa Prado e Archibald 2014 (PRADO; OLIVEIRA, 2015) obtiveram uma média de 2,58 e com a média geral das empresas privadas brasileiras, que tiveram uma avaliação 2,64, fica evidente a necessidade de implementação de um plano de crescimento, visto que esses valores dentro da escala de cinco pontos são modestos e têm uma avaliação apenas regular, conforme mostra o Gráfico 5.

Gráfico 5 - Análise comparativa da Avaliação Final de Maturidade

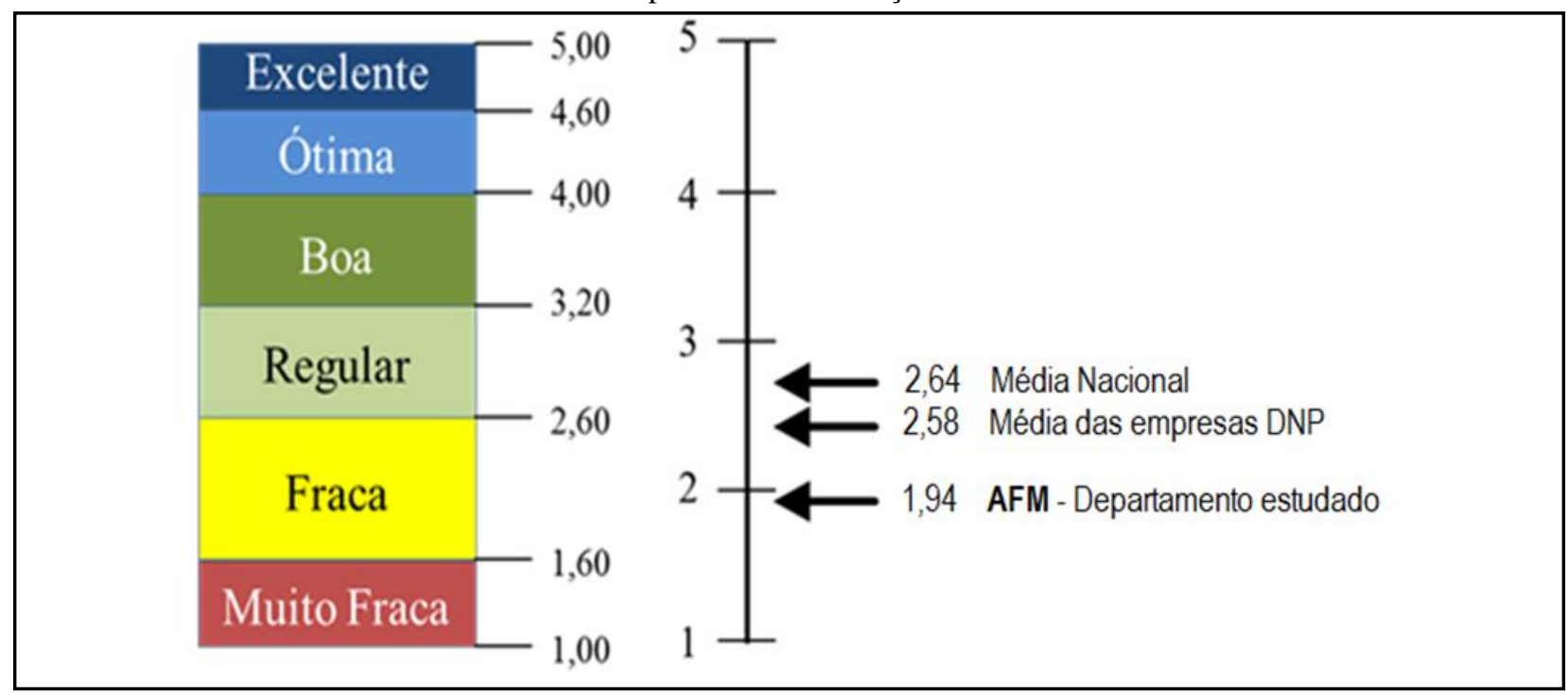

Segundo Prado e Fernandes (2015), as empresas de desenvolvimento de produtos (DNP e P\&D), classificadas no nível 1 têm um percentual de fracasso de $17 \%$, com estouro de custo da 
ordem de $20 \%$. Isso mostra a necessidade de se estabelecer um plano de crescimento para aumentar os índices de sucesso.

Ao considerar os resultados da análise de aderências às dimensões, apresentados no item 4.2.2, um plano de crescimento deve ser iniciado, com o estabelecimento de um programa de treinamento para melhorar as competências em gerenciamento de projetos, no desenvolvimento de uma metodologia e informatização dos processos.

\section{Considerações Finais}

Este estudo demonstrou que o método Prazo-MMGP pode ser aplicado em diferentes tipos organizações para avaliar o nível de maturidade de departamento e identificar os níveis de aderência às dimensões, entretanto é fortemente dependente do nível de conhecimento dos participantes quanto às práticas de gerenciamento de projetos do departamento.

Prado (2010) recomenda que o questionário deva ser respondido por um profissional que conheça o setor e suas práticas de gerenciamento de projetos, como o chefe do departamento, o coordenador do Escritório de Gestão de Projetos (EGP) ou algum auxiliar direto das funções citadas.

Isso ficou bastante evidente na análise estratificada por grupos de profissionais, separando aqueles que têm funções relacionadas ao gerenciamento dos projetos daqueles com foco principal na coordenação técnica. Foi possível também identificar que a qualidade dos resultados da pesquisa depende do nível de conhecimento dos participantes com relação a:

- Práticas de gerenciamento de projetos: conceitos, técnicas ferramentas e boas práticas (PMBOK, IPMA, entre outros);

- Conhecimento das práticas do departamento: é necessário um conhecimento amplo de todo o processo e ferramentas utilizadas, desde a fase de concepção, análise de viabilidade até o encerramento do projeto;

- Conhecimento da gestão da organização: conhecimento da estrutura de gestão da organização, pois alguns processos relacionados à gestão dos projetos ocorrem fora do âmbito do departamento; e

- Conhecimento do método MMGP: é fundamental que os participantes tenham um bom conhecimento do método e dos critérios de avaliação para cada nível de maturidade.

Este estudo demostrou que é possível utilizar a análise documental dos projetos para auxiliar no processo de análise dos resultados da pesquisa MMGP, trazendo detalhes específicos a partir de outra fonte de evidências que podem corroborar os resultados pesquisa ou indicar a necessidade de um maior aprofundamento das investigações nos casos onde há divergência. 
Desta forma, a análise documental pode contribuir para melhorar a avaliação do nível de maturidade nos casos onde os documentos relacionados ao gerenciamento dos projetos sejam disponibilizados para este fim.

\begin{abstract}
Projects are present in the daily lives of almost all organizations, they cover a wide range of investments representing nearly a third of world GDP. Many companies have been using maturity models to assess and establish actions to improve effectiveness in managing their projects. PradoMMGP Model has long been used in Brazil for the assessment of individual departments. This model, as well as most of them, is based solely on responses to a questionnaire, this may reduce the accuracy of the assessment results in situations where there is a very small amount of people qualified to answer it. This study aims to verify the applicability of the analysis of documents related to project management as a tool complementary to Prado-MMGP method to enlarge the discussions and improve the assessment of the level of maturity. It was decided to perform a single case study of descriptive-exploratory type in a new product development department of a midsize technology-based company. Using similar criteria to MMGP model could be calculated the adherence rates to the levels and dimensions of this model, which produced comparable results to those of the questionnaire application, which allowed analyzing the results by different sources of evidence. Thus, it was possible to note that the use of documentary evidence can bring additional information and help to improve the accuracy in the assessment of the level of maturity. However, its application is restricted to cases where the researcher has access to projects documentation.
\end{abstract}

Key-words: project management; maturity model; prado-MMGP model.

\title{
Referências
}

ARCHIBALD, R.D.; PRADO, D.S. Maturity by Project Category Model - MPCM. In: Archibald \& Prado. 2014. Disponível em: <http://www.maturityresearch.com>. Acesso em: 25/08/2014.

CARTWRIGHT, C.; YINGER, M. Project management competence development framework. In: PMI Global Congress, Budapest - Hungary, 2007. Disponível em: 〈http://www.pmi.org>. Acesso em 30/08/2014.

CARVALHO, M.M.; LAURINDO, F.J.B.; PESSÔA, M.S.P. Information technology project management to achieve efficiency in brazilian companies. In: KAMEL, Sherif. (Org.). Managing globally with information technology. Hershey: Idea Group, 2003. crossref

CARVALHO, M.M.; RABECHINI, R. Fundamentos em gestão de projetos: construindo competências para gerenciar projetos. 3. ed. São Paulo: Atlas, 2011.

CARVALHO, M. M., et al. Equivalência e completeza: análise de dois modelos de maturidade em gestão de projetos. In: Revista de Administração da USP. São Paulo: USP, jul./ago./set. 2005., v.40, n.3, p.289-300.

HERKENHOFF, D.A.; FIGUEIREDO, M.A.D; LIMA, G.B. Proposta unificadora de níveis de maturidade em gerenciamento de projetos. In: Anais eletrônicos do VII Congresso Nacional de Excelência em Gestão. Rio de Janeiro, 2011. Disponível em: <http://www.excelenciaemgestao.org/pt/edicoes-anteriores.aspx>. Acesso em: $30 / 08 / 2014$.

KERZNER, H. Strategic planning for project management using a project management maturity model. New York, USA: John Wiley \& Sons. Inc, 2001.

KERZNER, H. Project management: a systems approach to planning, scheduling, and controlling. 8. ed. New Jersey, USA: John Wiley \& Sons. Inc, 2009.

MAXIMIANO, A.C.A. Administração de projetos. 5. ed. São Paulo: Atlas, 2014. 
MEZZENA, B.; ZWICKER, R. Benefícios e dificuldades do modelo CMM de melhoria do processo de software. In: Revista de Gestão USP. São Paulo, v.14, n.3, p.107-121, julho/setembro. 2007.

PROJECT MANAGEMENT INSTITUTE (PMI). A Guide to the Project Management Body of Knowledge (PMBOK). Project Management Institute. 5.ed. Newton Square. Pennsylvania. U.S.A.: PMI, 2013a.

.Organizational Project Management Maturity Model(OPM3). Project Management Institute.3.ed. Newton Square. Pennsylvania. U.S.A.: PMI, 2013 b.

PMSURVEY. Estudo de benchmarking em gerenciamento de projetos 2008. Project Management Institute Chapters Brasileiros, 2008.

Brasileiros, 2009

Estudo de benchmarking em gerenciamento de projetos 2009.Project Management Institute - Chapters

PMSURVEY.PMSURVEY.ORG 2013 Edition. Project Management Institute Chapters, 2013.Disponível em: < http://www.pmsurvey.org>. Acesso em 30/08/2014.

PRADO, D. Maturidade em gerenciamento de projetos. INDG Tec. S.2.ed. Nova Lima, 2010.

PRADO, D.; FERNANDES, F.L. Pesquisa Archibald \& Prado: relatório DNP e P\&D, 2014 -indicadores, 2015. Disponível em: < http://www.maturityresearch.com>. Acesso em 19/02/2015.

PRADO, D.; OLIVEIRA, W. Pesquisa Archibald \& Prado: relatório geral 2014-2015. Disponível em: < http://www.maturityresearch.com>. Acesso em 19/02/2015.

SANTOS, L.G.C. Análise da influência da evolução na maturidade em gerenciamento de projetos no desempenho dos projetos. 2009. 145f. Dissertação (Mestrado) - Escola Politécnica da Universidade de São Paulo. Departamento de Engenharia Naval e Oceânica, São Paulo, 2009.

STANDISH GROUP. CHAOS manifesto 2013: think big, act small. Boston, 2013. Disponível em: <www.versionone.com/assets/ img/files/CHAOSManifesto2013.pdf>. Acesso em 10/09/2014.

THIELMANN, R.; SILVA, C. H. Avaliação de maturidade em gestão de projetos: estudo de caso de um projeto automotivo. In: GEPROS. Gestão da Produção, Operações e Sistemas, Bauru, Ano 9, nº 2, abr.-jun./2014, p. 35-49. crossref

VALLE, A.B.; SOARES, C.A.P.; FINOCCHIO Jr., J.; SILVA L.S.F. Fundamentos do gerenciamento de projetos. FGV. 2. ed. Rio de Janeiro, 2010.

VARGAS, R.V. Gerenciamento de projetos: estabelecendo diferenciais competitivos. Brasport. 6. ed. Rio de Janeiro, 2005. 


\section{Dados dos autores:}

Nome completo: Pérsio Vitor de Sena Abrahão

Filiação institucional: Universidade de Taubaté - UNITAU

Função ou cargo ocupado: Mestre em Gestão e Desenvolvimento Regional - Professor do MBA em Gerência de Projetos.

Endereço completo para correspondência: Av. Heitor Villa Lobos, 624 - Ap. 131-B, Vila Ema, São José dos Campos - SP, CEP 12243-260.

Telefones para contato: (12) 981463946

e-mail: persio.abrahão@terra.com.br

Nome completo: Edson Aparecida de Araújo Querido Oliveira

Filiação institucional: Universidade de Taubaté - UNITAU

Função ou cargo ocupado: Doutor em Organização Industrial - ITA - Professor do Mestrado em Gestão e Desenvolvimento Regional - Universidade de Taubaté - UNITAU (www.unitau.br).

Endereço completo para correspondência: Rua Expedicionário Ernesto Pereira, Portão 3, TaubatéSP CEP: 12030-320.

Telefones para contato: (12) 36218523

e-mail:edson.oliveira@unitau.com.br

Submetido em: 25-10-2015

Aceito em: 15-06-2016 